\title{
Experimental and numerical investigation of strain distribution of notched lead fatigue test specimen
}

\author{
Audun Johanson ${ }^{1, *}$, Luigi Mario Viespoli ${ }^{2}$, Bård Nyhus $^{3}$, Antonio Alvaro ${ }^{3}$, and Filippo Berto ${ }^{2}$ \\ ${ }^{1}$ Nexans Norway, Innspurten 9, 0663 Oslo, Norway \\ ${ }^{2}$ Department of Mechanical and Industrial Engineering, Norwegian University of Science and Technology (NTNU), Norway \\ ${ }^{3}$ Sintef Materials and Chemistry, Richard Birkelands vei 2B, 7031, Trondheim, NORWAY
}

\begin{abstract}
The work here presented focuses on the test methodology related to effect of stress concentrators in strain controlled structures. Cable sheathing as used in subsea power cables are investigated by cyclic fatigue testing, Digital Image Correlation (DIC) and 3D Finite Element Analysis. Focus is put on the strain distribution in conventional specimen geometries and under the presence of artificial notches. It is evident that standardized fatigue testing provides limited input to the final fatigue life of strain controlled power cable sheathing both accounting for expected and unintended stress concentrators. The limitations can be explained by measured strain distribution inherent in most fatigue test specimens. The use of DIC and 3D FEM provides valuable insight into both the theoretical and practical stress and strain distribution. This can help in understanding and overcoming geometrical test constraints, when compared to the actual component loading mode.
\end{abstract}

\section{Background and Scope}

Lead alloys sheathing is used as water barrier on high voltage subsea power cable. Subsea power cables are subjected to various cyclic loads during manufacturing, installation and operation. Cyclic loading of cable sheathing falls in the typical high cycle regime, but under global strain control in combination with nonlinear and time dependent material response.

Cable sheathing design and manufacturing is associated with multiple discontinuities which intensify the local stress and strain field. Such discontinuities might be inherent design features such as intimate contact with various strips such as transversal steel armoring, or local defects associated with the sheathing extrusion process such as indigenous oxides or intermetallic, or exogenous particles. Studies of such effects and fatigue properties of power cable sheathing is limited and rarely provide suitable input for fatigue life calculations of power cable sheathing where appropriate loading mode and design features are taken into account [1].

Discontinuities in components subjected to mechanical loading will cause concentration(s) in the local stress and strain field, and shorten the fatigue life of the component. Such effect is dependent on the material in question, particularly inelastic and relaxation properties as well as work hardening. These are associated with lead alloys [2]. Otherwise said, both the monotonic and the cyclic behavior of lead alloys exhibit a significant strain rate dependence. Effects of discontinuities are therefore not equivalent at any strain rate.

Often lab scale testing regarding such effects are done by implementing a well-defined notch detail on the test specimen. Due to the aforementioned material characteristics, the interpretation of results thereof is non-trivial as the component loading mode with respect to the detail-of-interest is not necessarily reproduced in smaller scale. Additional complications arise from the fact that the lab specimen geometry introduce deformation incompatibility zones, such as specimen edges and fillet radiuses, that do not exist for the real component which put into question the direct lab testing results transferability to the real component case. In this case, both the distribution of strain and strain rate must be carefully accounted for to ensure the suitability of the lab scale results. The following work aims to investigate the strain distribution and fatigue failure of notched test specimens extracted from cable sheathing.

\section{Material and experimental procedure}

\subsection{Materials}

The test material is extracted from as- manufactured power cable where the tubular lead sheathing was extruded from raw material according to PB012K in EN 12548. The chemical composition is reported in Table 1 . The procedure has been adopted to account for the presence of the defect in the lab specimen. A $0.5 \times 0.5 \times$

Corresponding author: audun.johanson@nexans.com 
0.4 sheathing thickness notch was made by electro discharge machining (EDM) on the convex surface of the curved specimen (Fig. 1). The design of the notch/discontinuity has been made based a potential surface penetrating inclusion originating from the extrusion process. Due to the small thickness of the sheathing $(3.3 \mathrm{~mm})$, the sheathing curvature in the transversal section has been kept for later fatigue testing in order to prevent buckling in the compressive phase and thus increase the maximum achievable strain range, as well as limiting the induced work hardening when preparing the sample (Fig. 2).

Table 1. Chemical composition of lead alloy PB012K

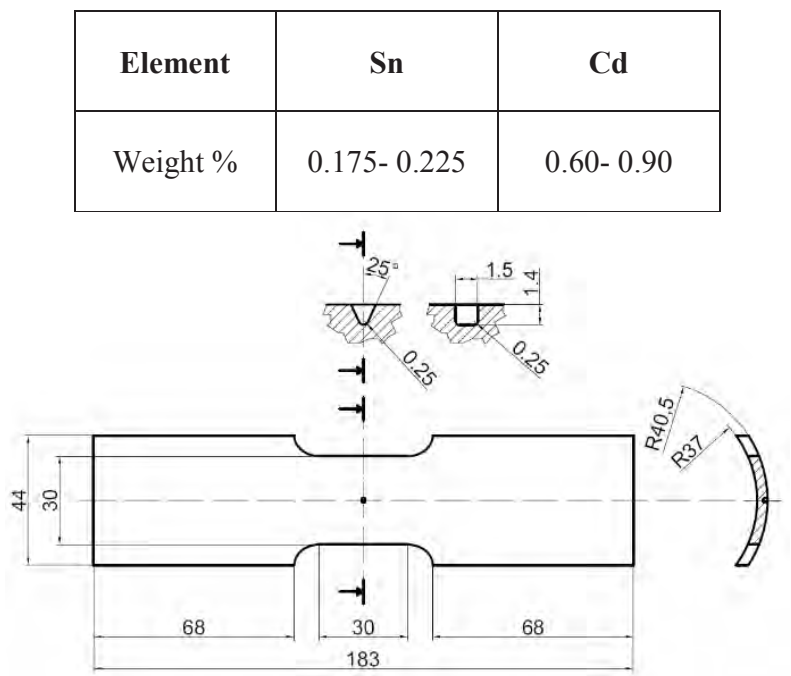

Fig. 1. Nominal dimensions of the specimens and of the notch detail.
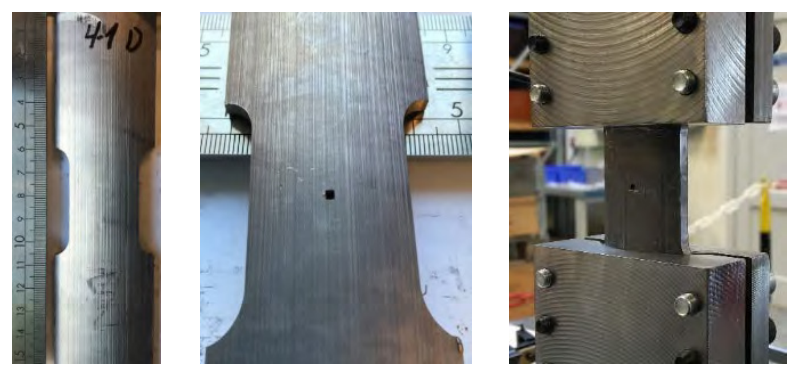

Fig. 2. The pictures show the sample un-notched (left), notched (middle) and in the custom-made, curved clamping system (right).

\subsection{Fatigue test procedure}

The effect of the discontinuity has been investigated by comparing cyclic tension-compression fatigue testing directed towards reversed bending of a power cables.

The tests have been run in position control, moving the head between two set points, which have been related to the strain range target values $(0.15 \%$ or $0.28 \%)$ prior to the test controlled by a mechanical extensometer. The extensometer was removed after the set-point definition due to sample indents (which where polished away). The recorded positions obtained before each test, in average corresponded to 0.008 and $0.0041 \mathrm{~mm}$ for a strain of
$0.28 \%$ and $0.15 \%$, respectively, has been kept constant during the complete the test duration. An appreciable decrease in the nominal amplitude was found for notched specimens Fig. 3, suggesting the presence of the notch affects the global response of the specimen.

For two of the tests at higher strain amplitude, the extensometer was applied on both sides of the specimen in order to verify whether or not an out-of-plane deformation occurred. The fatigue tests were performed following a frequency stepping up $(0.5,1,2$ and then 5 or $10 \mathrm{~Hz}$, according to the target frequency). During the test, load and displacement were recorded every 100 cycles and the number of cycles to failure was considered at the moment in which the tensile load fell below $0.2 \mathrm{kN}$, This was the highest lad value at which a through-thickness growing crack was visually detectable.

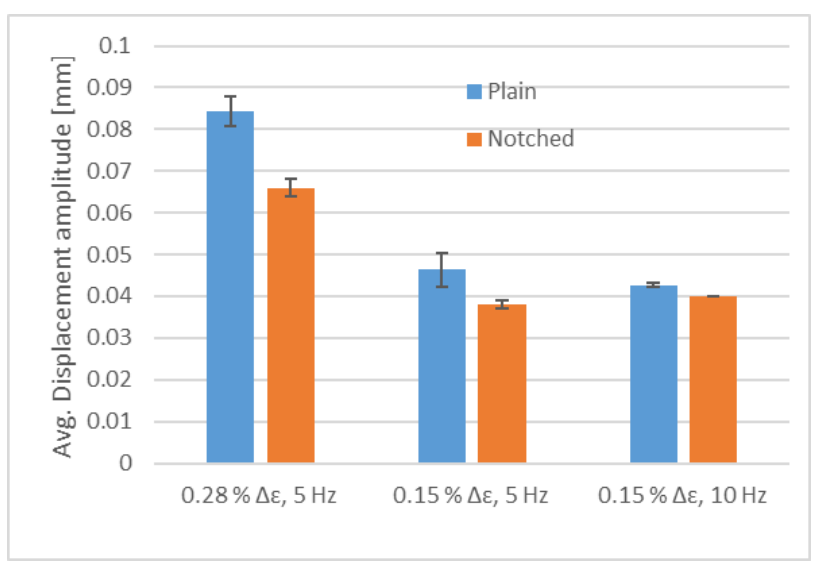

Fig. 3. Average displacement amplitude for plain and notched specimens.

\subsection{Strain rate dependency}

For lead alloys tested under displacement control, relaxation will cause a strong strain rate dependency. A series of tensile tests at various strain rates were conducted to identify the onset of appreciable strain rate dependency and thereby a common material law for finite element modelling. Tensile test where performed on dog- bone specimens (Fig. 4).

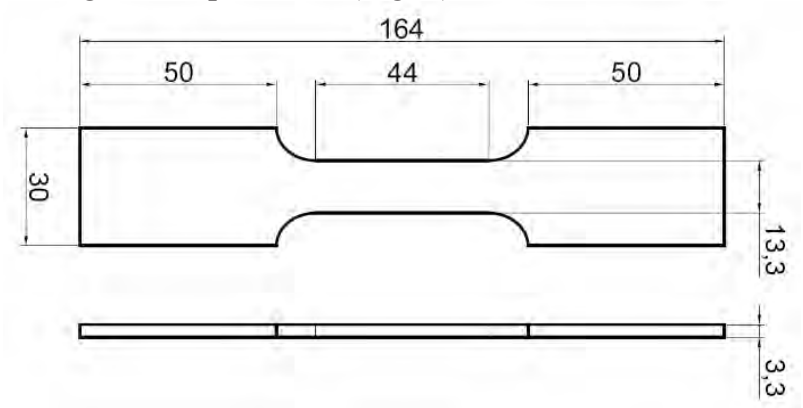

Fig. 4. Geometry of flat dog- bone tensile specimens.

The Stress-Strain curves obtained are shown in Fig. 5. It is evident how, for strain rates higher than $10^{-4} \mathrm{~s}^{-1}$, the effect of creep at room temperature is negligible, especially in the range of interest for the fatigue testing (up to $0.28 \%$ of nominal strain). Subsequently, the 
Digital Image correlation (DIC) material characterization on the flat dog-bone samples in Fig. 4 and the DIC analysis on the curved samples in Fig. 7 , have been performed at a nominal strain rate of $10^{-3} \mathrm{~s}^{-1}$.

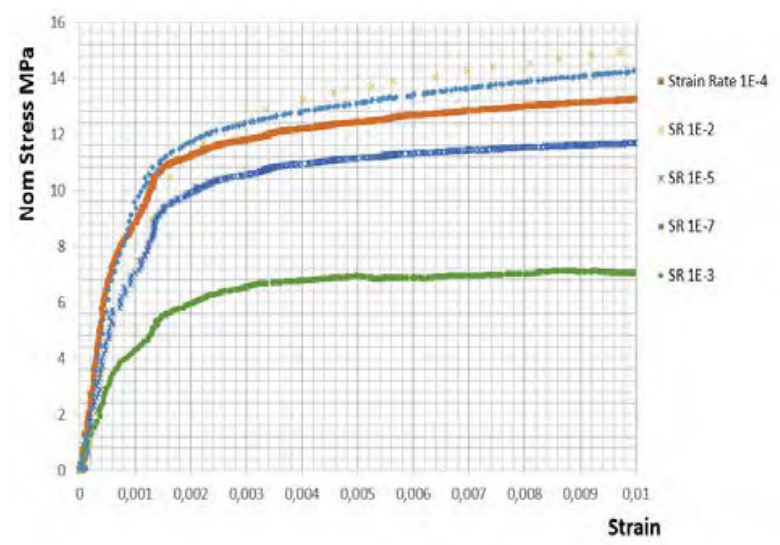

Fig. 5. Nominal Stress vs Strain curves for tensile dog-bone samples of lead alloy at different Nominal Strain Rates. At a rate higher than $10^{-4} \mathrm{~s}^{-1}$ the creeping effects are negligible within the cyclic strain range to-be investigated.

\subsection{Strain distribution}

The stress distribution in the specimen during the fatigue testing in relation to the failure location and final fatigue life from the fatigue testing is investigated by 3 dimensional finite element analysis (FEM) where monotonic tensile testing under DIC is used to validate the calculated strain distribution. Speckle pattern where applied on both flat dog-bone specimens and curved dog-bone specimens (Fig. 7) which were tested in a servo-hydraulic Zwick machine. A nominal strain rate of $10^{-3} \mathrm{~s}^{-1}$ where applied which assumes to constitute the lower bound strain rate below which relaxation/ creep effects takes effect (Fig. 5); in other words, above such strain rate value the material strain rate sensitivity is negligible.
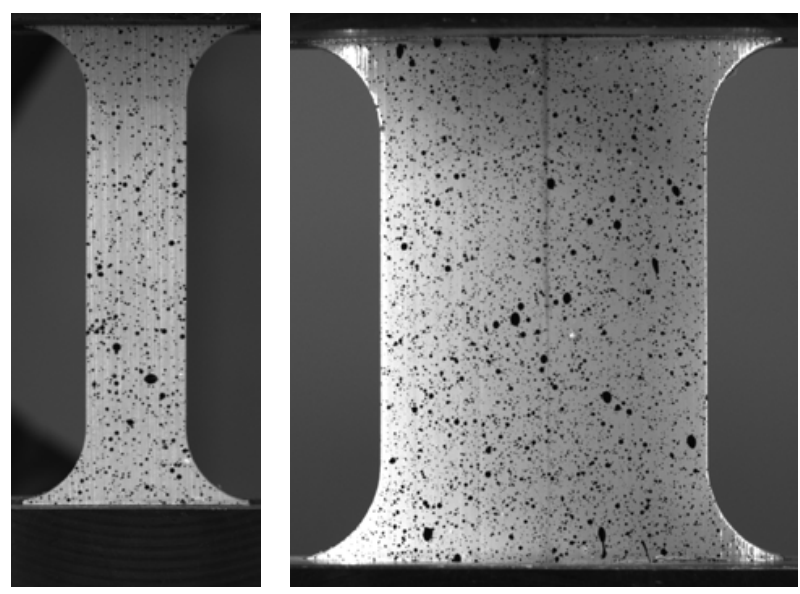

Fig. 7. A frame of the DIC images acquired for the flat tensile dog-bone (left) and of the curved specimen, un-notched, of the same geometry used for the fatigue testing (right).

\section{Experimental results}

\subsection{Fatigue tests}

Fatigue testing have been carried out at frequencies 5 and $10 \mathrm{~Hz}$ : the test results are summarized in the plot in Fig. 6. Result at the higher strain range, i.e. $0.28 \%$ show practically no difference in terms of fatigue life due to the presence of the notch. For the test performed at 0.15 $\%$ and $5 \mathrm{~Hz}$, the presence of the notch infers a decrease in the average fatigue life of about $65 \%$, i.e. from an average value of 536,000 cycles to 181,000 cycles. However, by stepping up the frequency from 5 to $10 \mathrm{~Hz}$, the detrimental effect induced by the presence of the notch seems to be reduced as in fact is rather the average fatigue life of the un-notched specimens to drop of about $40 \%$, from 536,000 to an average of 323,000 cycles. No significant difference is found for the notched specimens.

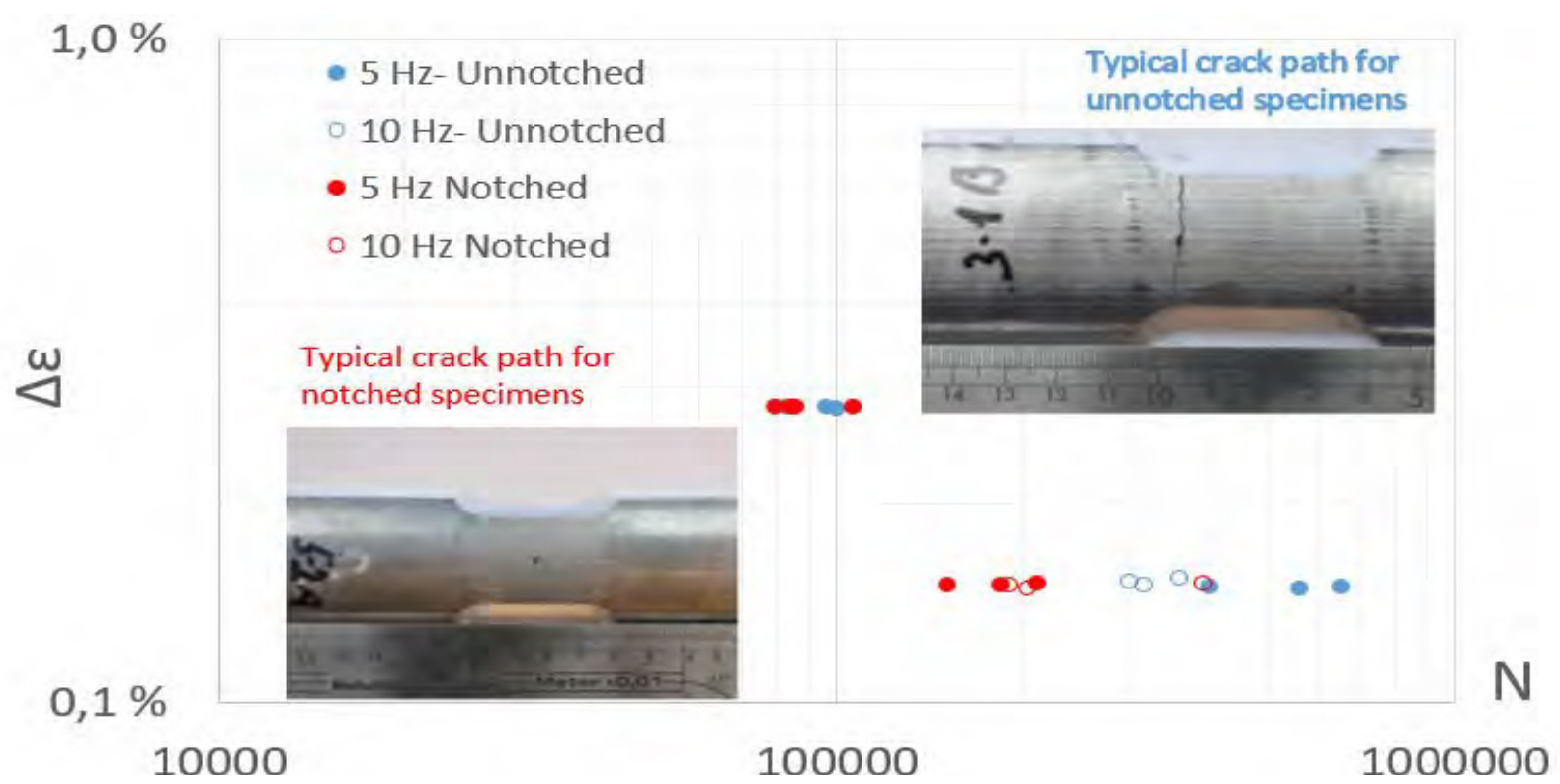

Fig. 6. Fatigue test results together with typical crack paths. It was found that for the lower strain range tests $(0.15 \%)$ notched specimens, the failure consistently initiated from the specimen edge at the height/ position of the notch (Lower left picture). When the notch was not present, failure tended to initiate from the fillet edge. For $0.28 \%$ strain range, both notched and unnotched tended to fail from the middle (notch- height) region. 
Post mortem analysis of the specimens also reveals a difference when it comes to the crack location. In 7/9 un-notched specimens the crack initiated from the specimen fillet radius, 8/10 notched specimens failed by the crack initiating from the outer edge, but in correspondence of the notch height/ location (middle region of the sample). Note that $2 / 3$ unnotched $0.28 \%$ strain range samples failed from the middle edge position. i.e. the trend more evident for the $0.15 \%$ strain range samples. This indicates that the presence of the notch induces a variation in the stress/strain field strong enough to alter the position of the initiation point but still the specimen edge constitutes the "weakest" point when it comes to either crack initiation or crack- initiation and growth. The investigation of this effect and the primary aim for continued DIC and FEM analysis reported in the following paragraphs.

\subsection{Strain distribution}

The strain distribution in the different regions of the specimen (Fig. 8) together with the resulting stress-strain plot obtained through post-processing of the DIC images.

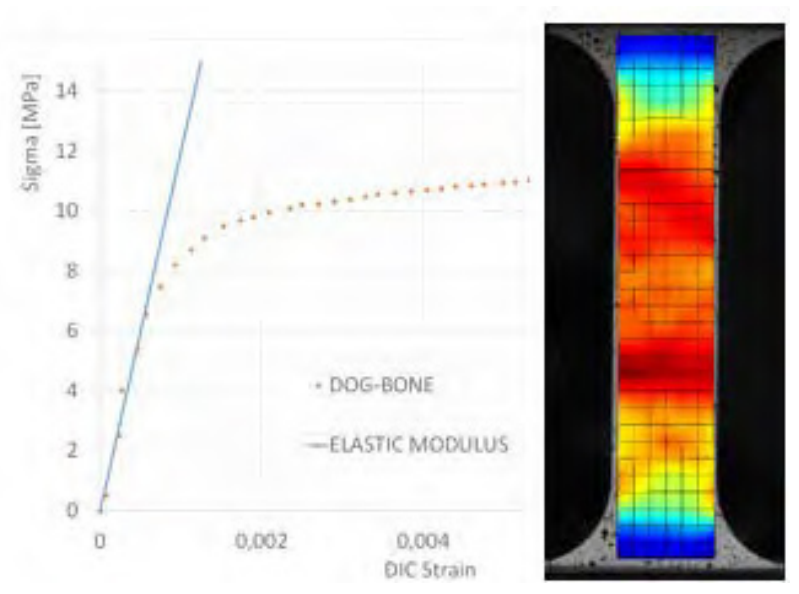

Fig. 8. Obtained tensile curve and strain distribution as acquired by DIC.

Tensile testing under DIC has permitted to record the true Stress-Strain characteristic of the alloy. This provides the material constitutive behaviour for the elastic-plastic FEM analysis on both the un-notched and the notched sample.

\section{Finite element analysis}

The aim of the analysis is to give basis for a better understanding of the influence of the notch on the failure dynamics and to eventually reproduce numerically the behavior of the alloy. The comparison between the DIC and FEM analysis is executed at the same nominal/global strain, i.e. measured from the displacement of the boundary divided by the free length in the FEM analysis.
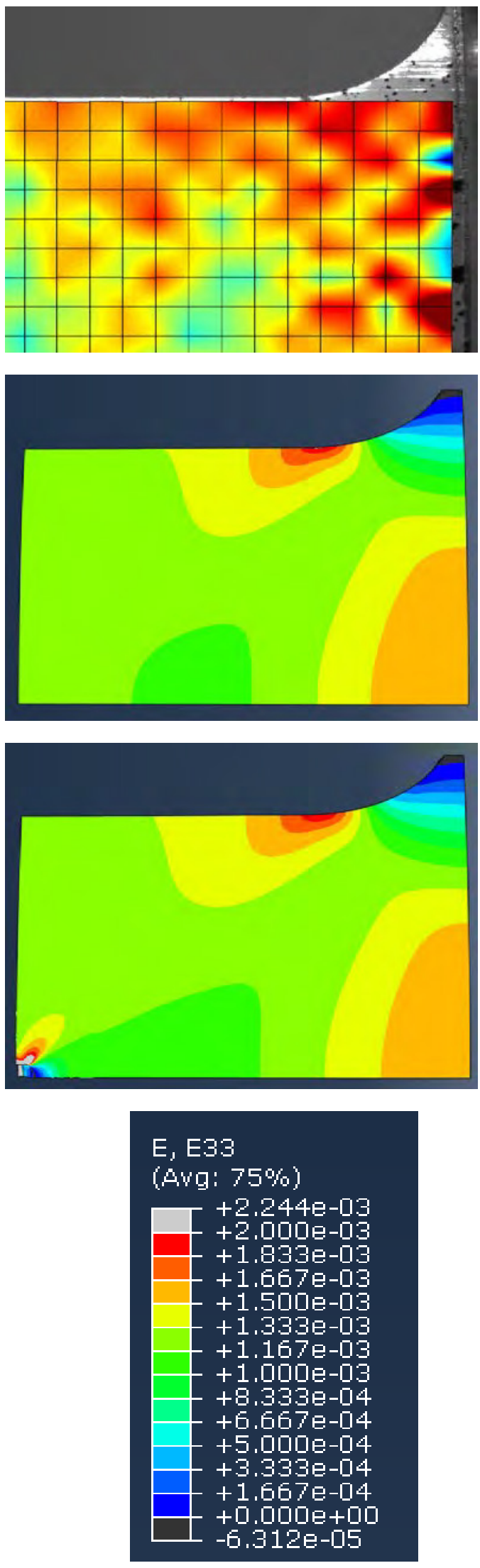

Fig. 9. Strain field along the longitudinal direction of a quarter of the un-notched specimen, DIC (top) and FEM (2. from the top). In figure (3. from the top), the same strain field for the notched specimen. The scale adopted for the strain is the same for all captures (lower). All strain field corresponds to a nominal strain of $1.1 \mathrm{E}^{-3}$. 
For the test, the real border effects and eventual deformation of the sample close to the clamping area does not allow precise strain estimation. Therefore, the nominal strain is obtained from DIC on a vector which covers the full length of the sample. The evaluation of the equivalent nominal strain is then made by considering the FEM analysis. The results obtained show, at the same equivalent loading condition and in the same scale, that the FEM analysis is feasible to reproduce the strain field in magnitude and shape (Fig. 9, Fig. 10), with a concentration of the strain for the unnotched sample in correspondence of the fillet radius and the clamping area.

The results of the FEM analysis on the notched geometry shows a much higher strain, at comparable equivalent boundary displacement, which justifies the final fracture passing through the notch, but there is no evidence from the numerical stress field of the reason why fatigue fractures in the notched specimens have been observed to initiate on the edge of the sample, at the height of the notch, but not from the notch itself.
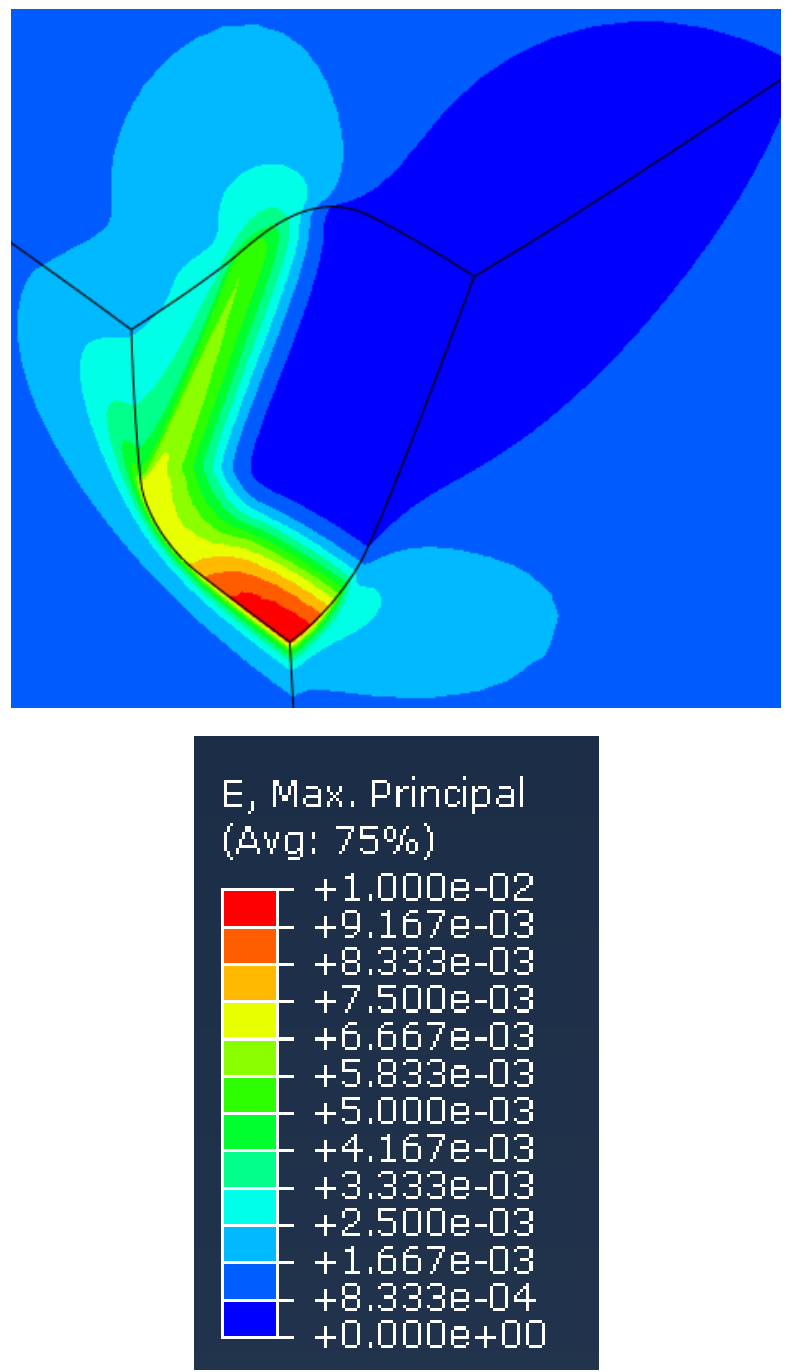

Fig. 10. Maximum principal strain in the notch, at the same nominal strain of the captures in Fig. 9. The scale shows a notable strain intensification due to the notch.

\section{Conclusion and further work}

It is evident from the fatigue test results that the impact of the notch is higher for low strain ranges. This relation appears to be dependent on test frequency. However, the imposed test frequencies imply a strain rate exceeding appreciable creep. Understanding of the impact of frequency, particularly taking into account the expected fatigue life reduction due to creep is critical and the subject of ongoing work. Location of failure initiation also supports a different effect of the notch for the two tested strain range levels, where high cyclic strain range fail from the middle region of the test specimens both with and without a notch. For lower cyclic strain range, failure only occur in the middle region when the notch is present. The Numerical calculations and tensile test experiments under DIC show that the strain concentration on specimen fillets is significantly higher than the nominal strain range and supports failure from this location. Numerical calculations show that the notched detail is associated with the highest strain concentration which justifies both the reduction in fatigue life and change in failure location. However, as the final crack do not tend to initiate at notch the test results appear inconsistent with the analysis.

As the lead alloy is expected to undergo irreversible deformation at the imposed strain ranges, hardening and local blunting can be mechanisms that cause redistribution of the strain and differentiates the monotonic and cyclic strain fields thus explains a change in failure initiation. However: no evidence of blunting or major changes of the notch geometry has been observed after fatigue testing. Regardless, the material law used in the numerical study as well as the DIC strain is obtained from monotonic cases. The cyclic material response will differ. Ongoing work is put into the development of a dynamic material law and in obtaining the DIC recording to have a quantification of the strain distribution variation and development during the actual fatigue test. The inconsistently between local strain concentration and failure initiation can also be related to the crack initiation and growth dynamics- meaning that the strain field must not only favor crack initiation but continued growth.

Finally, it should be noted that failure initiation from the specimen edge is a test artifact with respect to the final application as such details are absent for tubular components. Crack initiation from the sample edge indicates that even if the relative effect of a notchedunnotched component is larger than for the notched unnotched test specimen, the laboratory scale fatigue test result for both notched and unnotched samples are conservative compared to the real component. Despite of this, care should be taken in assessing fatigue life ductile components such as power cable sheathing, by standardized testing. 
The work supports the use of numerical calculations coupled with experimental validations using DIC to both interpret fatigue results and design fatigue test specimens. This is critical for accurate fatigue life predications of component subjected to cyclic loadingparticularly in the presence of local details and strain distribution.

\section{Acknowledgment}

The authors wish to thank NEXANS Norway and the Research Council of Norway for the financial support through ENERGIX Programme, Contract No.256367/E20.

\section{References}

1. International Lead Zinc Research Organization, Inc. (ILZRO), "Program on Technology Innovation: Grain Growthin Lead Alloy Sheathin and its Influence on the Life of Lead-Sheathed Power Cables, " EPRI, Palo Alto, Ca, (2007).

2. S. Guruswamy, Engineering Properties and Applications of Lead Alloys, New York: Marcel Dekker, (2000). 